\title{
The effect of physical activity across the menstrual cycle on reproductive function
}

\author{
Katherine A. Ahrens, $\mathrm{PhD}^{\mathrm{a}}$, Catherine J. Vladutiu, $\mathrm{PhD}^{\mathrm{b}}$, Sunni L. Mumford, PhDa, Karen C. \\ Schliep, PhD ${ }^{\mathrm{a}}$, Neil J. Perkins, $\mathrm{PhD}^{\mathrm{a}}$, Jean Wactawski-Wende, $\mathrm{PhD}^{\mathrm{c}}$, and Enrique F. \\ Schisterman, PhD $^{\mathrm{a}}$ \\ aDivision of Intramural Population Health Research, Eunice Kennedy Shriver National Institute of \\ Child Health \& Human Development, 6100 Executive Blvd, National Institutes of Health, \\ Bethesda, Maryland 20892 \\ bDepartment of Epidemiology, Gillings School of Global Public Health, 137 E. Franklin Street, CB \\ \#8050, University of North Carolina, Chapel Hill, North Carolina 27599-8050 \\ 'Department of Social and Preventive Medicine, 270 Farber Hall, University at Buffalo, Buffalo, \\ New York 14214
}

\section{Abstract \\ Purpose-To evaluate the association between physical activity (PA) across the menstrual cycle and reproductive function.}

\begin{abstract}
Methods-The BioCycle Study (2005-2007) followed 259, healthy premenopausal women not using hormonal contraceptives for up to two menstrual cycles ( $\mathrm{N}=509$ cycles). Serum leptin, estradiol, progesterone, luteinizing hormone, follicle-stimulating hormone, and testosterone were measured five to eight times per cycle. Linear mixed models were used to estimate the effect of past-week PA (measured four times during each cycle) on hormone levels. Past-week PA was categorized into tertiles based on metabolic equivalent [MET]-h/week (cut-points were 15.3 and 35.7). Risk ratios for sporadic anovulation were estimated using generalized linear models. Analyses adjusted for habitual PA (assessed at baseline), body mass index, race, age, and perceived stress. Linear mixed models used inverse probability weights to control for concurrent reproductive hormones and caloric intake.
\end{abstract}

Results-High past-week PA was inversely associated with leptin $(-6.6 \%, 95 \%$ confidence interval $[-10.6,-2.5])$ and luteal phase progesterone $(-22.1 \%[-36.2,-4.7])$ as compared with low past-week PA. High past-week PA was not significantly associated with sporadic anovulation (adjusted risk ratio=1.5 [0.6, 3.4]).

\footnotetext{
(c) 2013 Elsevier Inc. All rights reserved.

Corresponding Author's and Request for Reprints Contact Information: Enrique Schisterman, Ph. D., Eunice Kennedy Shriver National Institute of Child Health and Human Development, NIH, MSC 7510, 6100 Executive Blvd., 7B03, Bethesda, MD 20892-7510, Tel: 301-435-6893, Fax: 301-402-2084. Author responsible for correspondence related to the manuscript: Katherine Ahrens, Ph.D., Eunice Kennedy Shriver National Institute of Child Health and Human Development, NIH, MSC 7510, 6100 Executive Blvd., 7B03, Bethesda, MD 20892-7510, Tel: 301-594-9149, Fax: 301-402-2084, katherine.ahrens@ nih.gov.

Conflicts of interest

We declare we have no conflicts of interest.

Publisher's Disclaimer: This is a PDF file of an unedited manuscript that has been accepted for publication. As a service to our customers we are providing this early version of the manuscript. The manuscript will undergo copyediting, typesetting, and review of the resulting proof before it is published in its final citable form. Please note that during the production process errors may be discovered which could affect the content, and all legal disclaimers that apply to the journal pertain.
} 
Conclusions-High levels of PA were modestly associated with changes in select hormones, but not sporadic anovulation among moderate to highly active premenopausal women.

\section{MeSH}

hormones; leisure activity; menstrual cycle; motor activity; women

\section{INTRODUCTION}

Physical activity (PA) is beneficial for women's health as it is associated with a decreased risk of cardiovascular disease; breast and colon cancers; type 2 diabetes; osteoporosis; and other adverse health outcomes. [1] The U.S. Department of Health \& Human Services recommends that individuals engage in at least 150 minutes of moderate-intensity PA per week in order to gain these important health benefits. [1] As the majority $(\sim 70 \%)$ of reproductive-age women report meeting these guidelines, [2] it is important to understand the effects of PA on reproductive hormones and ovulation, which can subsequently influence fertility outcomes.

There is evidence suggesting that high-intensity activity is associated with menstrual dysfunction and subfertility among high-performance female athletes. [3] Previous studies have found that high-intensity activity is associated with amenorrhea, oligomenorrhea, luteal phase deficiency, and anovulation, likely through disturbances of the hypothalamicpituitary-adrenal axis. [3, 4] It has been hypothesized that suppression of gonadotropinreleasing hormone resulting from exercise-associated hypothalamic dysfunction can delay menarche and disrupt menstrual cycle patterns by limiting the secretion of luteinizing hormone (LH) and follicle-stimulating hormone (FSH). [3, 5]

While much is known about PA and reproductive function among athletes, less is known about this association among more moderately active women. Several studies have examined the association between moderate levels of PA and menstrual cycle characteristics [6-8] or urinary and salivary hormone concentrations. [9-12] However, fewer studies have assessed the effect of PA on circulating hormone concentrations, [13-15] and they were limited by assessing PA at only one time point and collecting blood at most two times across a menstrual cycle.

The primary objective of our study was to examine the association between PA and reproductive hormones, including estradiol, progesterone, LH, FSH, testosterone, and leptin, across the menstrual cycle. Our secondary objective was to examine the association between PA and the risk of sporadic anovulation. The effects of PA on reproductive function have important implications for fecundability and for modifying chronic disease risk across a woman's lifespan.

\section{METHODS}

\section{Study population}

The BioCycle Study, conducted in 2005-2007, was a cohort study of 259 regularly menstruating, healthy premenopausal women from Western New York who were followed over one $(n=9)$ or two $(n=250)$ menstrual cycles. Women were ineligible for the study if they used oral contraceptives or medications for a chronic medical condition; were recently pregnant or breastfeeding; had been diagnosed with a menstrual or ovulatory disorder; planned to consume a restricted diet in the next 3 months or self-reported their body mass index (BMI) as less than 18 or greater than $35 \mathrm{~kg} / \mathrm{m}^{2}$ at screening. Additional information about the study population is described elsewhere. [16] Among the 449 women screened for 
study participation, 319 met the eligibility criteria, 276 enrolled in the study, and 17 withdrew after enrollment. The University at Buffalo Health Sciences Institutional Review Board (IRB) approved the study and served as the IRB designated by the National Institutes of Health for this study under a reliance agreement. All participants provided written informed consent.

\section{Measures}

\section{Reproductive hormones, anovulation and menstrual cycle characteristics-}

Women provided morning fasting blood samples five to eight times per cycle. Fertility monitors (Clearblue Easy Fertility Monitor; Inverness Medical, Waltham, Massachusetts) were used to time mid-cycle visits, with the remaining visits scheduled according to an algorithm that considered each woman's typical cycle length. [17] For a standardized 28-day menstrual cycle, blood samples were collected during the following expected phases: menses (standardized day 2), follicular (standardized day 7), ovulatory (standardized days 12, 13, and 14), and luteal (standardized days 18, 22, and 27). Most women adhered to the study protocol with $94 \%$ providing at least seven blood samples per cycle. Samples $(\mathrm{N}=3,903)$ were processed according to standard protocols and frozen at $-80^{\circ} \mathrm{C}$ within 90 minutes of phlebotomy. [18] They were then shipped on dry ice to analytical laboratories. Samples from each participant were batched together and measured consecutively to limit analytical variability.

Serum estradiol, progesterone, LH, and FSH levels were measured by solid-phase competitive chemiluminescent enzymatic immunoassays on the DPC Immulite 2000 analyzer (Siemens Medical Solutions Diagnostics, Deerfield, Illinois) at Kaleida Laboratories in Buffalo, New York. Serum total testosterone was measured by liquid chromatography/tandem mass spectrometry using the Shimadzu Prominence Liquid Chromatogram with an ABSceix 5500 tandem mass spectrometer at the Advanced Research and Diagnostics Laboratory (Minneapolis, Minnesota). Increased sensitivity was achieved by using $100 \%$ acetronitrile mobile phase B as the solvent gradient elution and adding a low standard of $4 \mathrm{ng} / \mathrm{dL}$. Serum leptin was measured by immunoassay using the Mercodia Leptin ELISA (Mercodia AB, Uppsala, Sweden) by the Advanced Research and Diagnostics Laboratory (see above). The interassay coefficients of variation were: $<10 \%$ for estradiol; < $14 \%$ for progesterone; < $4 \%$ for $\mathrm{LH}$ and $\mathrm{FSH}$; and < $7 \%$ for testosterone. For leptin, select batches of measurements were recalibrated post-assay by a calibration curve estimated from all the calibration data. [19] The interassay coefficient of variation for leptin was $<10.2 \%$ after recalibration. Values falling below the limit of detection (LOD) for each assay were rare $(<3 \%)$ and were replaced with values equal to the LOD divided by the square root of two. [20] All hormones were logarithmically transformed for normality.

Anovulatory cycles $(n=42)$ were defined as cycles with a peak progesterone concentration $\leq$ $5 \mathrm{ng} / \mathrm{mL}$ and no observed serum LH peak among samples collected during the latter half of the cycle (i.e. days 22 and 27), which could indicate a missed luteal phase serum sample. [21] Menstrual cycle characteristics (menstrual cycle length, follicular and luteal phase length, menses length, and total blood loss during menses) were determined from fertility monitor data, serum hormone levels, daily diaries, and pictograms for documenting total blood loss. [21, 22]

Physical activity-There were three measures of self-reported PA: habitual PA (measured at baseline using the long-form International Physical Activity Questionnaire, IPAQ [23, 24]); past-week PA (measured on days 2, 7, 14, and 22 using the short-form IPAQ, IPAQ$\mathrm{SF}$ ); and daily vigorous PA (measured using a daily diary). Both IPAQs assessed the duration and frequency of walking, moderate activity, and vigorous activity lasting for at 
least 10 minutes during the previous week, with the long-form IPAQ including additional questions about specific types of activity. Total MET-hours per week (MET-h/week) were calculated by summing the individual MET-h/week for each activity according to published guidelines. [24] Past-week PA was further classified using the MET-h/week spent in vigorous activity only and termed "vigorous past-week PA." For daily vigorous PA, participants were instructed to record the amount of time spent each day engaged in vigorous activity, defined as "activities that take hard physical effort and make you breathe much harder than normal."

For the measure of habitual PA, women were categorized into tertiles based on MET-h/week reported. For average past-week PA, the MET-h/week from the IPAQ-SFs were averaged across each cycle and across the entire study period for each individual and then categorized into tertiles, respectively. For the time-varying analyses, past-week PA was categorized into tertiles based on the distribution of MET-h/week reported at each assessment, thus a woman could be categorized as having high past-week PA at one assessment and low past-week PA at another. In addition, tertile cut-points varied by assessment day as the distribution of $\mathrm{MET}$-h/week varied. Daily vigorous activity for the day prior to serum collection was dichotomized as any versus none.

Covariates-Self-administered questionnaires were used to assess demographics, smoking behavior, and perceived stress (measured by the 14-item Cohen Perceived Stress Scale) [25] at study enrollment. BMI was calculated using weight and height as measured by trained personnel at the baseline visit. Total caloric intake was assessed by four 24-hour dietary recalls measured during each cycle.

Statistical analysis-Participant characteristics were compared by tertile of average pastweek PA across the study period with Fisher's exact and ANOVA tests used to evaluate differences. For repeated measures within individual women (i.e., from multiple cycles and days), unadjusted linear mixed models were used to calculate means and assess differences across PA tertiles for continuous variables and unadjusted generalized linear models were used to assess differences for categorical variables. Unadjusted linear mixed models were also used to assess differences in hormone concentrations among PA tertiles by standardized serum collection day. The correlation structure was specified as unstructured for days and first-order autoregressive for cycles.

The association between PA and reproductive hormone concentrations was estimated using four PA exposure definitions: 1) habitual PA; 2) past-week PA; 3) vigorous past-week PA; and 4) daily vigorous PA. Linear mixed models with random intercepts were used to assess the effect of each PA definition on estradiol, progesterone, LH, FSH, testosterone, and leptin levels across the menstrual cycle while accounting for variation in baseline hormone levels between women. PA exposures that varied over the cycle were treated as time-varying exposures in the analysis. In all four exposure models, 477 (12\%) of the 3903 serum samples were excluded due to observations missing data on perceived stress $(n=15)$, time-varying PA $(n=354)$, or caloric intake $(n=111)$. Models were performed using all available observations for estradiol, FSH, testosterone, and leptin; only observations during the luteal and ovulatory visits were assessed for progesterone and $\mathrm{LH}$, respectively, because of their greatest variation during these phases. Models were adjusted for age, BMI, race, and perceived stress. Models of time-varying PA were also adjusted for habitual PA to examine acute PA effects on hormone changes. Results of the models were presented as average percent change in nontransformed reproductive hormone values relative to the lowest PA using the following formula: $\left.\left\{\left(\exp ^{\wedge} \beta\right)-1\right] \times 100 \%\right\}$. 
For the time-varying analyses, we also estimated parameters using weighted models to control for time-varying factors, such as caloric intake and reproductive hormones, which could be both causes and consequences of time-varying PA. [26] We constructed inverse probability weights using concurrent estradiol, progesterone, FSH, leptin, and caloric intake measurements. These weights had a mean value of 1.00 with a range from 0.59 to 2.16.

The association between PA and sporadic anovulation was estimated using four PA exposure definitions: 1) habitual PA; 2) past-week PA during the first half of the cycle; 3 ) vigorous past-week PA during the first half of the cycle and 4) daily vigorous PA during the first half of the cycle. Risk ratios (RR) were estimated using generalized linear models, while adjusting for age, BMI, race, and perceived stress. For past-week and daily vigorous PA, models were further adjusted for habitual PA and only included PA assessed prior to the expected time of ovulation to preserve the proper temporal ordering of the exposureoutcome relationship.

Bias analysis-For the association between time-varying past-week PA and estradiol concentrations, error due to misclassification of MET-h/week was quantified using probabilistic bias analysis. [27] We utilized a regression equation previously published from a Women's Health Initiative validation study that compared self-reported activity-related energy expenditure with that from doubly-labeled water in 450 postmenopausal women. [28] We used the validation study's regression coefficients to calculate the correctly classified MET-h/week for our study participants based on their age, race, BMI and selfreported MET-h/week. Our study data was simulated 5000 times, with regression coefficients selected by Monte Carlo sampling techniques to draw from a normal distribution. [27, 29] Adjusted linear mixed regression models were applied to each simulated dataset and sampling errors were incorporated (Rothman, Greenland, and Lash, Modern Epidemiology III, page 366 equation 19-17). The $2.5^{\text {th }}, 50^{\text {th }}$ and $97.5^{\text {th }}$ percentiles of all simulations were reported.

\section{RESULTS}

\section{Study population}

Two-hundred and fifty nine women were followed for one $(n=9)$ or two $(n=250)$ menstrual cycles. For women followed for two menstrual cycles, the majority had consecutive cycles $(92 \% ; n=229)$ and the remaining 21 women had cycles that were, on average, two months apart (mean $=55$, range 31 to 134 days). The average age of participants was 27.3 years (standard deviation [SD], 8.2) and average BMI was 24.1 (SD, 3.9) (Table 1). Habitual and daily vigorous PA varied by average past-week PA, with higher levels of activity in the highest tertile of past-week PA; no other differences were observed.

The median past-week PA (averaged for each individual across the study) was 25 MET-h/ week (range 0 to 301 ), which is equivalent to approximately 7.5 hours of walking or 3 hours of vigorous PA per week. Overall, past-week PA did not vary substantially across the menstrual cycle $(\mathrm{p}=0.08)$. However, PA was higher during the late luteal phase (assessed at day 2) compared with the early luteal phase (assessed at day 22) (median 25 vs. 19 MET-h/ week, respectively, $\mathrm{p}=0.01$ ).

\section{Menstrual cycle characteristics}

Average follicular phase lengths differed by past-week PA averaged within each cycle $(\mathrm{p}<0.01)$ with increasing lengths observed as PA increased (Table 2). No differences were observed for the other menstrual cycle characteristics or the proportion of cycles that were anovulatory. 


\section{Reproductive hormones}

Hormone concentrations across a standardized 28-day menstrual cycle by past-week PA averaged across each cycle are shown in Figure 1. Significant differences $(\mathrm{p}<0.05)$ were observed for progesterone (day 7: middle < highest PA tertile); LH (days 7, 14, 18, 22 : lowest < highest PA tertile); and leptin (days 2, 7, 13, 18, 27: lowest > highest tertile; days 7, 14, 18: middle > highest tertile).

The associations between PA and changes in hormone concentrations across the menstrual cycle are shown in Figure 2. Estradiol, ovulatory LH, and FSH concentrations were similar between the highest and lowest PA levels, regardless of PA exposure definition. Luteal progesterone was lower in the highest versus lowest PA levels for the three time-varying PA exposure definitions $(-22.1 \%$ for past-week PA [ $p=0.02],-28.9 \%$ for vigorous past-week PA [p<0.01], and $-17.6 \%$ for daily vigorous PA [p<0.01]). Testosterone concentrations were higher in the highest vigorous past-week PA tertile compared with the lowest tertile $(4.4 \%, \mathrm{p}<0.01)$. Leptin concentrations were lower in the highest versus lowest levels among the three time-varying PA assessments $(-6.6 \%$ for past-week PA [p<0.01], $-6.3 \%$ for vigorous past-week PA [p<0.01], and $-6.2 \%$ for daily vigorous $P A[p<0.01])$. The effects of the middle versus lowest tertile of PA were, for the most part, either attenuated compared with the highest PA level or imprecisely estimated (Supplementary Figure 1). Adjustment for time-varying concurrent hormone concentrations and caloric intake had little impact on the effect estimates, indicating that time-varying confounding by these factors was minimal (data not shown). Probabilistic bias analysis revealed that correction for misclassification of past-week PA resulted in a slightly stronger association between PA and estradiol, but the confidence interval was wide and crossed the null (bias-corrected percent change $=-3.4$, $95 \%$ simulation interval $[-13.6,10.1]$; conventionally adjusted percent change $=-2.9,95 \%$ confidence interval $[-9.4,4.0])$.

\section{Sporadic anovulation}

Cycles with high past-week PA during the first half of the cycle had a slightly higher risk of sporadic anovulation compared with low PA, but this difference was not statistically significant (Table 3). A similar finding was observed for daily vigorous PA during the first half of the cycle. In contrast, habitual PA showed a lower risk of anovulation among the highest PA tertile, but this confidence interval also crossed the null.

\section{DISCUSSION}

This study observed modest associations between time-varying PA and select reproductive hormones among a cohort of healthy, premenopausal women. Specifically, high levels of PA throughout the menstrual cycle were associated with lower concentrations of leptin and luteal progesterone. Habitual PA, measured once at baseline, did not appear to be associated with reproductive hormone concentrations. The risk of sporadic anovulation among this cohort of regularly menstruating women was not significantly affected by PA level.

Previous observational studies of PA and hormones among reproductive-aged women have found similar patterns for progesterone and leptin. In two multi-cycle studies, urinary progesterone was lower, especially during the luteal phase, in recreational runners (averaging 20 miles per week) and exercising women (averaging 7.5 hours of vigorous PA per week) compared with sedentary women. $[9,12]$ In addition, numerous studies have also found associations between acute increases in PA and decreases in leptin concentrations. [5, 30]

In contrast to previous studies, we found no association between PA and estradiol. Prior observational studies have found that high levels of PA were associated with approximately 
10-20\% lower urinary, $[9,11,12]$ salivary, $[10]$ and plasma estradiol concentrations compared with the lowest PA group with PA measured by a variety of methods and findings more pronounced for luteal phase estradiol. [13, 14] Experimental data also show decreased estradiol concentrations following a 5-month exercise intervention. [31] Lower cumulative estradiol concentrations have been proposed as an important mechanism for explaining the protective association between PA and premenopausal breast cancer. [32]

Explanations for the discrepancy between our null findings and the inverse associations observed for estradiol in previous studies are unclear, but could be due to differences in methodology. In our study, PA was primarily measured using the IPAQ, while other studies have used different questionnaire-based tools. Results from our post-hoc bias analysis showed that misclassification of PA could have accounted for some, but not all, of our null findings. Another explanation is that our study was restricted to regularly menstruating women, which could have limited the comparability of our study population to the ones previously examined. In fact, in these previous studies, attenuation of the PA-estradiol association was observed after excluding women with menstrual cycle irregularities. [11, 13] We also restricted our study to women with a BMI between 18 and 35; this likely enhanced our ability to control for this confounder by design rather than introduced bias, but could limit the generalizability of our findings.

This study expands on previous literature by examining the association between PA and reproductive function using a prospective time-varying analysis that controls for changes in concurrent hormones and caloric intake. Additional strengths of this study include the large size of the cohort (259 women); the enumeration of two menstrual cycles for most participants (97\%); serum measures of hormones at five to eight time points across the cycle, identified with the use of a fertility monitor; and the assessment of PA using both validated questionnaires and daily diaries. [23]

This study has several limitations. Bio-measurements of PA using accelerometers or doubly labeled water are more accurate than self-reported measures but were not used in this study. [33] Although habitual PA was measured at baseline, participants were instructed to recall PA over the previous 7 days, thus it may not reflect usual PA levels. Time-varying analyses model the effect of consistently being within a particular PA tertile over time rather than the effect within a defined group of women over time. This can limit the generalizability of the findings as they pertain more to a hypothetical behavior than an individual's actual behavior pattern. The regression coefficients used for our bias analysis were estimated from postmenopausal women and may have limited applicability to our premenopausal cohort, however similar data in premenopausal women were not available. Ultrasound technology could have provided a more accurate assessment of anovulatory cycles than our hormonebased definition. In addition, we limited our ability to detect the risk of chronic anovulation by excluding women with known ovulatory disorders and irregular menstrual cycle lengths.

In our cohort of healthy, moderate to highly active premenopausal women, PA did not appear to substantially change hormone levels during the menstrual cycle or significantly affect the risk of sporadic anovulation. These findings suggest that PA may not adversely affect fecundability and further reinforce the beneficial effect of PA on women's health.

\section{Supplementary Material}

Refer to Web version on PubMed Central for supplementary material.

\section{Acknowledgments}

Funding 
This work was supported by the Intramural Research Program of the Eunice Kennedy Shriver National Institute of Child Health and Human Development, National Institutes of Health (contract \# HHSN275200403394C).

We gratefully acknowledge the women who participated in the BioCycle Study for their extraordinary commitment to the study.

\section{List of abbreviations}

$\begin{array}{ll}\text { BMI } & \text { body mass index } \\ \text { CI } & \text { confidence interval } \\ \text { FSH } & \text { follicle-stimulating hormone } \\ \text { IPAQ } & \text { International Physical Activity Questionnaire } \\ \text { IRB } & \text { Institutional Review Board } \\ \text { LH } & \text { luteinizing hormone } \\ \text { MET-h/week } & \text { metabolic equivalent of task hours per week } \\ \text { PA } & \text { physical activity } \\ \text { RR } & \text { risk ratio } \\ \text { SD } & \text { standard deviation }\end{array}$

\section{References}

1. U.S. Department of Health \& Human Services. 2008 Physical Activity Guidelines for Americans. 2008

2. Centers for Disease Control and Prevention. Prevalence of self-reported physically active adults-United States, 2007. MMWR Morbidity and mortality weekly report. 2008; 57:1297-300. [PubMed: 19052527]

3. Warren MP, Perlroth NE. The effects of intense exercise on the female reproductive system. The Journal of Endocrinology. 2001; 170:3-11. [PubMed: 11431132]

4. Welt CK, Chan JL, Bullen J, Murphy R, Smith P, DePaoli AM, et al. Recombinant human leptin in women with hypothalamic amenorrhea. N Engl J Med. 2004; 351:987-97. [PubMed: 15342807]

5. Loucks AB, Thuma JR. Luteinizing hormone pulsatility is disrupted at a threshold of energy availability in regularly menstruating women. Journal of Clinical Endocrinology and Metabolism. 2003; 88:297-311. [PubMed: 12519869]

6. Harlow SD, Matanoski GM. The association between weight, physical activity, and stress and variation in the length of the menstrual cycle. American Journal of Epidemiology. 1991; 133:38-49. [PubMed: 1983897]

7. Sternfeld B, Jacobs MK, Quesenberry CP Jr, Gold EB, Sowers M. Physical activity and menstrual cycle characteristics in two prospective cohorts. American Journal of Epidemiology. 2002; 156:402-9. [PubMed: 12196309]

8. Cooper GS, Sandler DP, Whelan EA, Smith KR. Association of physical and behavioral characteristics with menstrual cycle patterns in women age 29-31 years. Epidemiology. 1996; 7:624-8. [PubMed: 8899389]

9. De Souza MJ, Miller BE, Loucks AB, Luciano AA, Pescatello LS, Campbell CG, et al. High frequency of luteal phase deficiency and anovulation in recreational women runners: blunted elevation in follicle-stimulating hormone observed during luteal-follicular transition. Journal of Clinical Endocrinology and Metabolism. 1998; 83:4220-32. [PubMed: 9851755]

10. Jasienska G, Ziomkiewicz A, Thune I, Lipson SF, Ellison PT. Habitual physical activity and estradiol levels in women of reproductive age. European journal of cancer prevention: the official journal of the European Cancer Prevention Organisation (ECP). 2006; 15:439-45. [PubMed: 16912573] 
11. Matthews CE, Fortner RT, Xu X, Hankinson SE, Eliassen AH, Ziegler RG. Association between physical activity and urinary estrogens and estrogen metabolites in premenopausal women. Journal of Clinical Endocrinology and Metabolism. 2012; 97:3724-33. [PubMed: 22855335]

12. De Souza MJ, Toombs RJ, Scheid JL, O’Donnell E, West SL, Williams NI. High prevalence of subtle and severe menstrual disturbances in exercising women: confirmation using daily hormone measures. Human reproduction. 2010; 25:491-503. [PubMed: 19945961]

13. Tworoger SS, Missmer SA, Eliassen AH, Barbieri RL, Dowsett M, Hankinson SE. Physical activity and inactivity in relation to sex hormone, prolactin, and insulin-like growth factor concentrations in premenopausal women - exercise and premenopausal hormones. Cancer Causes and Control. 2007; 18:743-52. [PubMed: 17549594]

14. Verkasalo PK, Thomas HV, Appleby PN, Davey GK, Key TJ. Circulating levels of sex hormones and their relation to risk factors for breast cancer: a cross-sectional study in 1092 pre- and postmenopausal women (United Kingdom). Cancer Causes and Control. 2001; 12:47-59. [PubMed: 11227925]

15. Nagata C, Kaneda N, Kabuto M, Shimizu H. Factors associated with serum levels of estradiol and sex hormone-binding globulin among premenopausal Japanese women. Environmental Health Perspectives. 1997; 105:994-7. [PubMed: 9300929]

16. Wactawski-Wende J, Schisterman EF, Hovey KM, Howards PP, Browne RW, Hediger M, et al. BioCycle study: design of the longitudinal study of the oxidative stress and hormone variation during the menstrual cycle. Paediatric and Perinatal Epidemiology. 2009; 23:171-84. [PubMed: 19159403]

17. Howards PP, Schisterman EF, Wactawski-Wende J, Reschke JE, Frazer AA, Hovey KM. Timing clinic visits to phases of the menstrual cycle by using a fertility monitor: the BioCycle Study. American Journal of Epidemiology. 2009; 169:105-12. [PubMed: 18974081]

18. Browne RW, Bloom MS, Schisterman EF, Wactawski-Wende J, Hovey K, Trevisan M, et al. Analytical and biological variation of F2-isoprostanes during the menstrual cycle. Clinical Chemistry. 2009; 55:1245-7. [PubMed: 19359531]

19. Whitcomb BW, Perkins NJ, Albert PS, Schisterman EF. Treatment of batch in the detection, calibration, and quantification of immunoassays in large-scale epidemiologic studies. Epidemiology. 2010; 21 (Suppl 4):S44-50. [PubMed: 21422966]

20. Hurnong R, Reed L. Estimation of average concentration in the presence of non-detectable values. Applied Occupational and Environmental Hygiene. 1990; 5:46-51.

21. Mumford SL, Steiner AZ, Pollack AZ, Perkins NJ, Filiberto AC, Albert PS, et al. The utility of menstrual cycle length as an indicator of cumulative hormonal exposure. Journal of Clinical Endocrinology and Metabolism. 2012; 97:E1871-9. [PubMed: 22837188]

22. Dasharathy SS, Mumford SL, Pollack AZ, Perkins NJ, Mattison DR, Wactawski-Wende J, et al. Menstrual bleeding patterns among regularly menstruating women. American Journal of Epidemiology. 2012; 175:536-45. [PubMed: 22350580]

23. Craig CL, Marshall AL, Sjöström M, Bauman AE, Booth ML, Ainsworth BE, et al. International physical activity questionnaire: 12-country reliability and validity. Med Sci Sports Exerc. 2003; 35:1381-95. [PubMed: 12900694]

24. Committee IR. Guidelines for Data Processing and Analysis of the International Physical Activity Questionnaire (IPAQ). Nov.2005

25. Cohen S, Kamarck T, Mermelstein R. A global measure of perceived stress. Journal of Health and Social Behavior. 1983; 24:385-96. [PubMed: 6668417]

26. Robins JM, Hernán MA, Brumback B. Marginal structural models and causal inference in epidemiology. Epidemiology. 2000; 11:550-60. [PubMed: 10955408]

27. Lash, T.; Fox, M.; Fink, A. Applying Quantitative Bias Analysis to Epidemiologic Data. Springer; 2009.

28. Neuhouser ML, Di C, Tinker LF, Thomson C, Sternfeld B, Mossavar-Rahmani Y, et al. Physical Activity Assessment: Biomarkers and Self-Report of Activity-Related Energy Expenditure in the WHI. Am J Epidemiol. 2013; 177:576-85. [PubMed: 23436896] 
29. Ahrens K, Lash TL, Louik C, Mitchell AA, Werler MM. Correcting for exposure misclassification using survival analysis with a time-varying exposure. Annals of Epidemiology. 2012; 22:799-806. [PubMed: 23041654]

30. Bouassida A, Chamari K, Zaouali M, Feki Y, Zbidi A, Tabka Z. Review on leptin and adiponectin responses and adaptations to acute and chronic exercise. British Journal of Sports Medicine. 2010; 44:620-30. [PubMed: 18927166]

31. Kossman DA, Williams NI, Domchek SM, Kurzer MS, Stopfer JE, Schmitz KH. Exercise lowers estrogen and progesterone levels in premenopausal women at high risk of breast cancer. Journal of Applied Physiology. 2011; 111:1687-93. [PubMed: 21903887]

32. Maruti SS, Willett WC, Feskanich D, Rosner B, Colditz GA. A prospective study of age-specific physical activity and premenopausal breast cancer. Journal of the National Cancer Institute. 2008; 100:728-37. [PubMed: 18477801]

33. Matthews CE, Moore SC, George SM, Sampson J, Bowles HR. Improving self-reports of active and sedentary behaviors in large epidemiologic studies. Exercise and Sports Science Review. 2012; 40:118-26. 
Estradiol

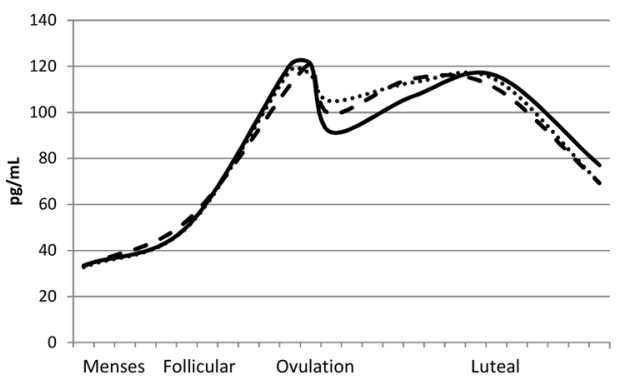

LH

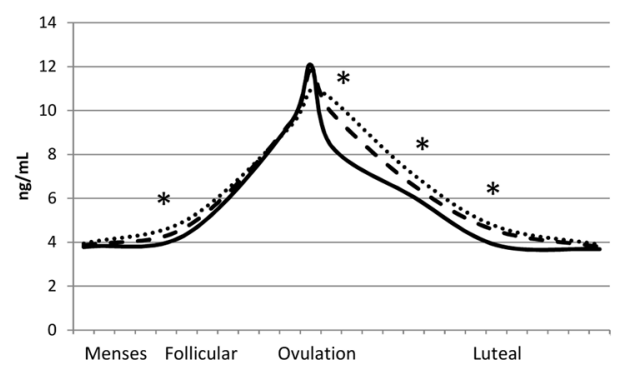

Testosterone

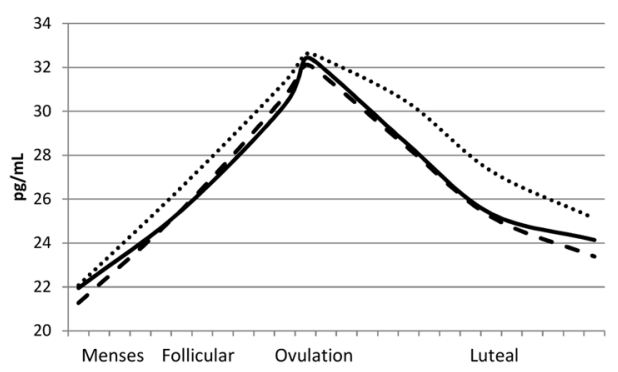

Progesterone

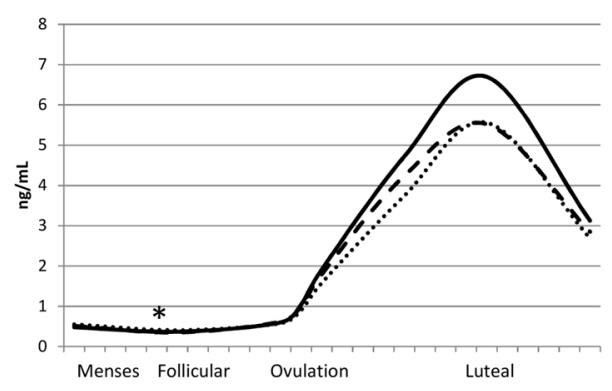

FSH

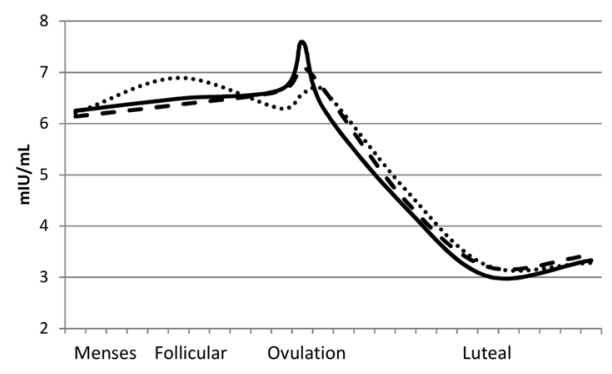

Leptin

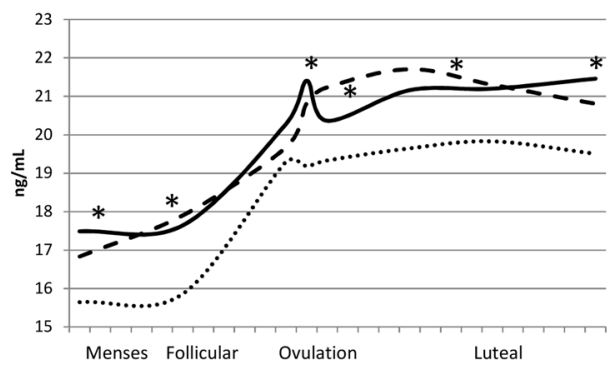

Figure 1.

Geometric mean hormone concentrations across a standardized 28-day menstrual cycle by average past-week physical activity within each cycle: lowest tertile (median=9, maximum =15 MET-h/week; solid line), middle tertile (median=24 MET-h/week; dashed line) and highest tertile (median=66, minimum=37 MET-h/week; dotted line).

$\mathrm{PA}=$ physical activity; $\mathrm{LH}=$ luteinizing hormone; $\mathrm{FSH}=$ follicle-stimulating hormone $* P$-value $<0.05$; geometric means were estimated and $p$-values were assessed using unadjusted linear mixed models. Significant differences were observed for progesterone (day 7: middle < highest tertile); LH (days 7, 14, 18, 22: lowest < highest tertile); and leptin (days 2, 7, 13, 18, 27: lowest > highest tertile; days 7, 14, 18: middle > highest tertile). 


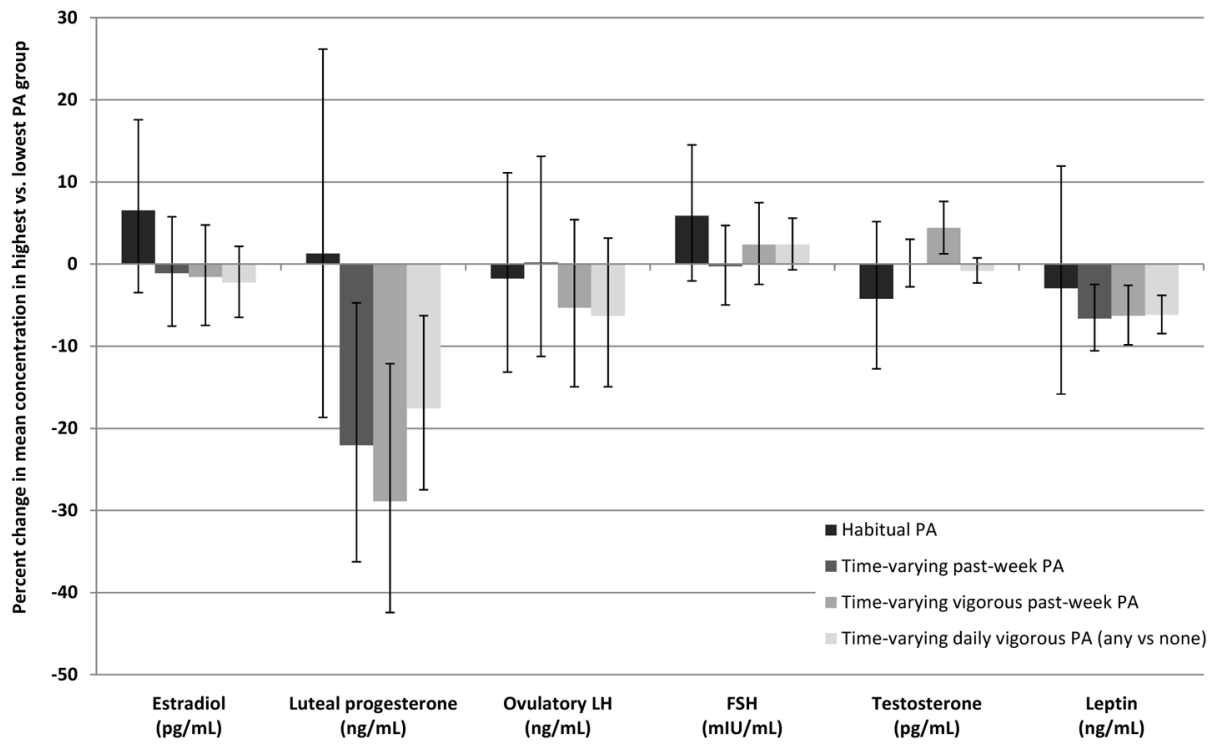

Figure 2.

The adjusted percent change in average hormone concentration for highest physical activity (PA) compared to lowest PA for four exposure definitions of PA: habitual PA, time-varying past-week PA, time-varying vigorous past-week PA and time-varying daily vigorous PA (any vs. none).

$\mathrm{LH}=$ luteinizing hormone; $\mathrm{FSH}=$ follicle stimulating hormone; $\mathrm{MET}=$ metabolic equivalent; PA= physical activity

The tertile cut-points were: 35.1 and 83.3 MET-h/week for habitual PA; 15.3 and 35.7 MET-h/week (on average) for time-varying past-week PA; and 1 and 15.5 MET-h/week (on average) for time-varying vigorous past-week PA. All analyses adjusted for baseline confounders; time-varying PA analyses were further adjusted for habitual PA and for concurrent hormones and caloric intake using inverse probability weights. 


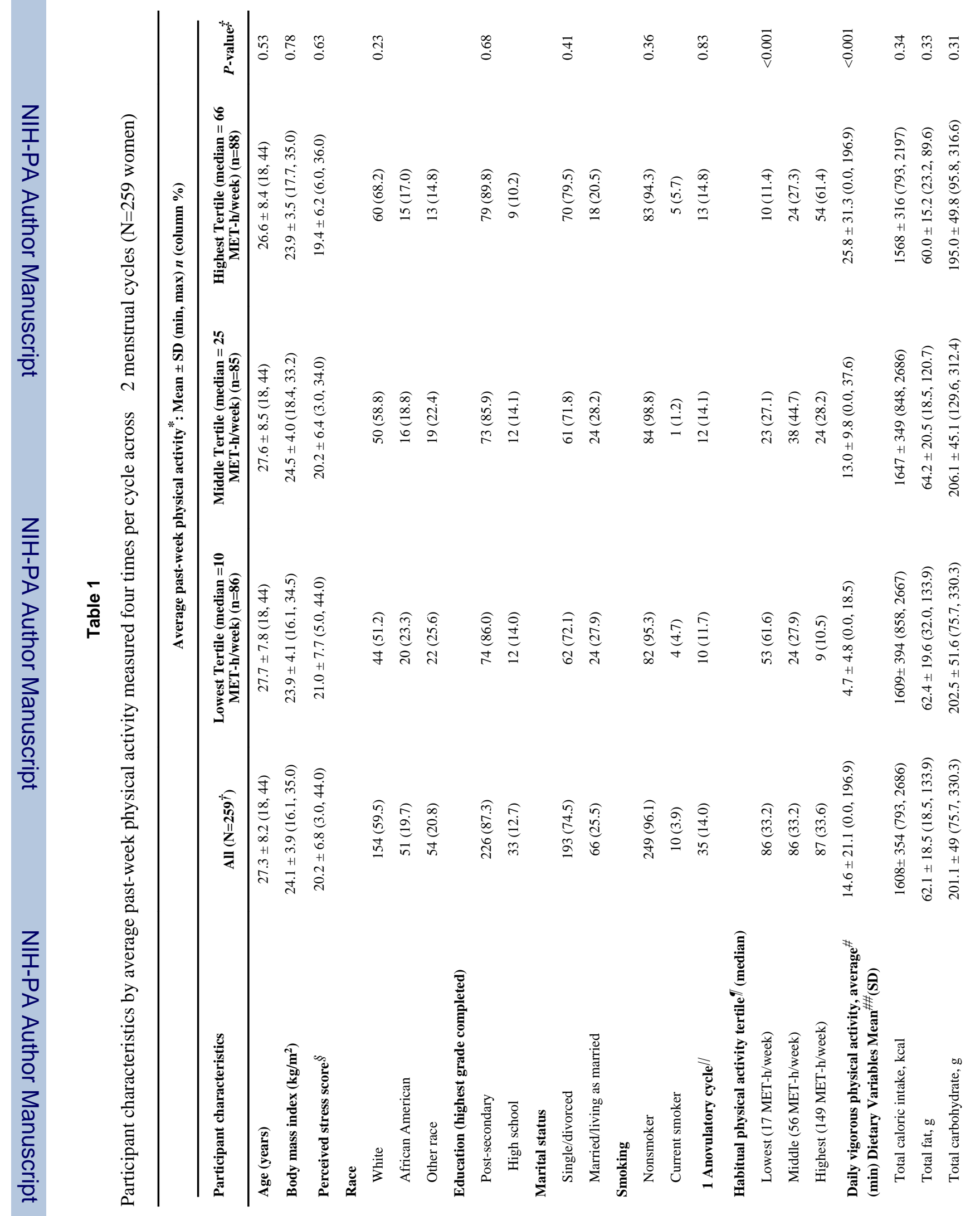




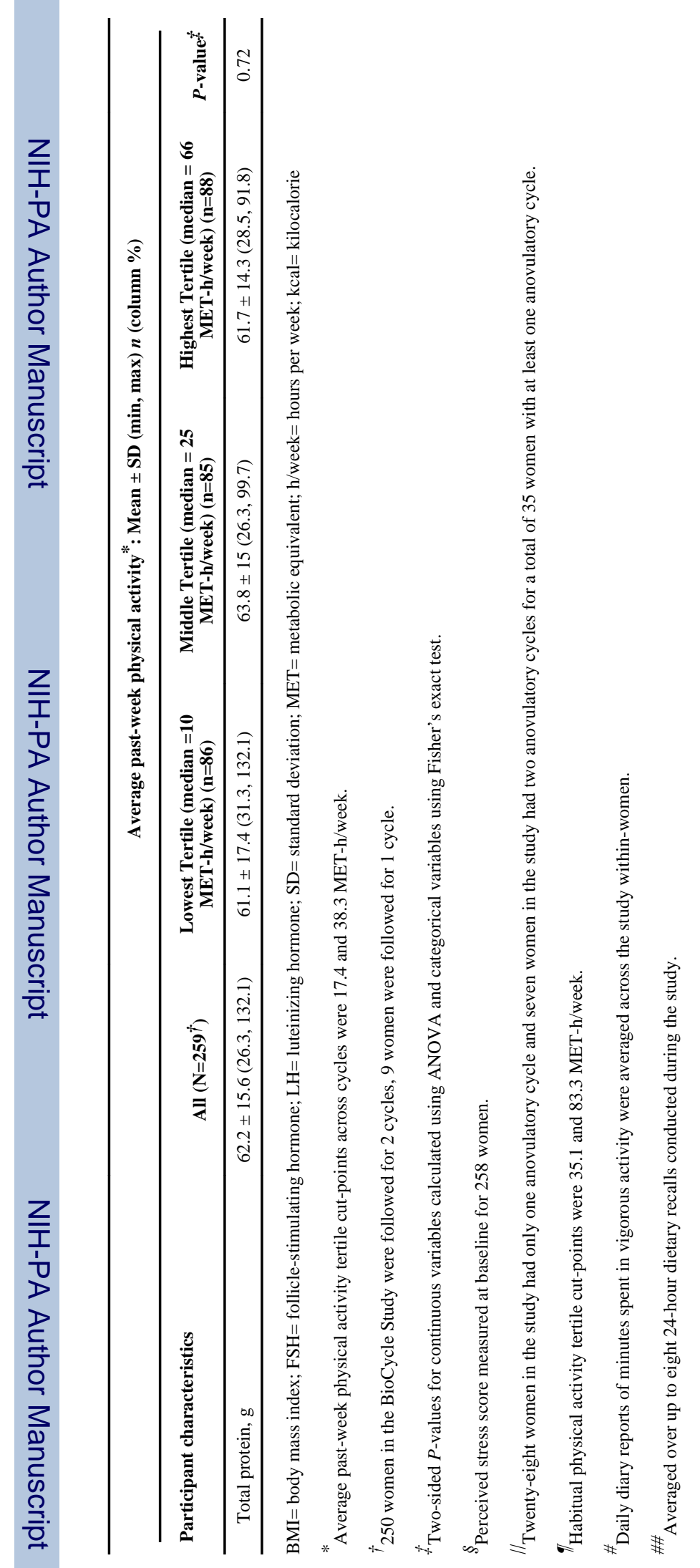

Ann Epidemiol. Author manuscript; available in PMC 2015 February 01. 


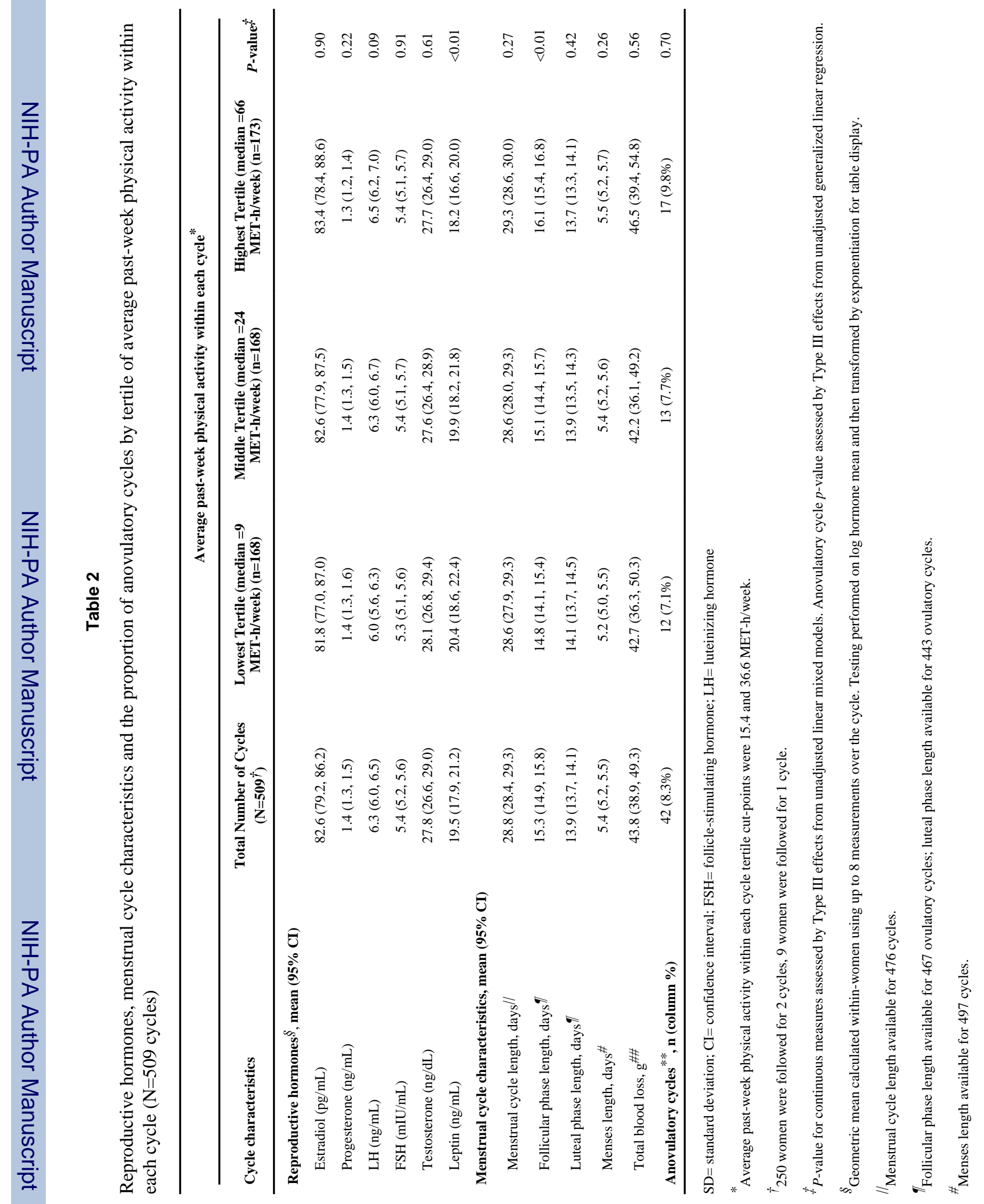




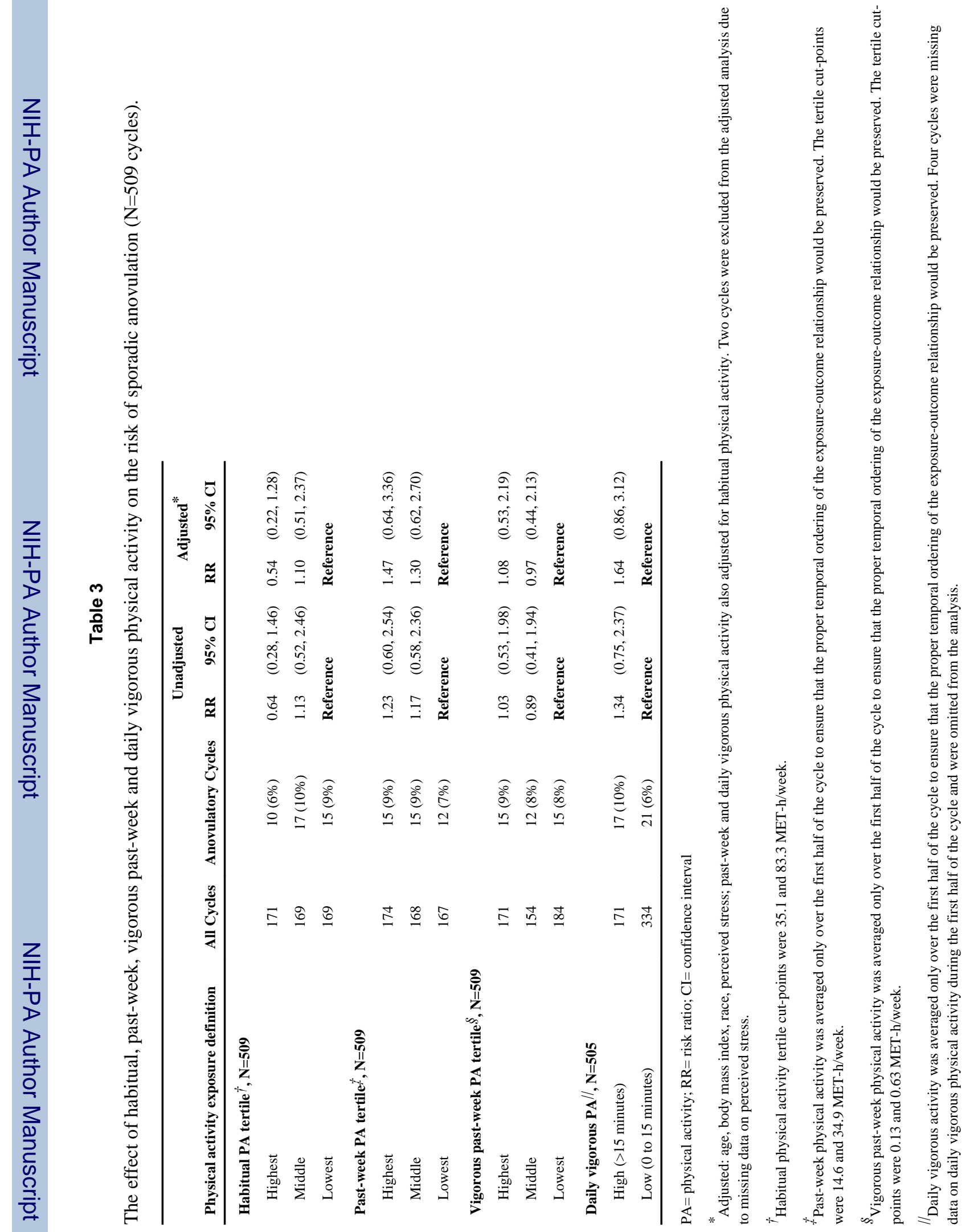

